\title{
Bases for Structures and Theories II
}

\author{
Jeffrey Ketland
}

\begin{abstract}
In Part I of this paper (Ketland in Logica Universalis 14:357$381,2020)$, I assumed we begin with a (relational) signature $P=\left\{P_{i}\right\}$ and the corresponding language $L_{P}$, and introduced the following notions: a definition system $d_{\Phi}$ for a set of new predicate symbols $Q_{i}$, given by a set $\Phi=\left\{\phi_{i}\right\}$ of defining $L_{P}$-formulas (these definitions have the form: $\left.\forall \bar{x}\left(Q_{i}(x) \leftrightarrow \phi_{i}\right)\right)$; a corresponding translation function $\tau_{\Phi}: L_{Q} \rightarrow$ $L_{P}$; the corresponding definitional image operator $D_{\Phi}$, applicable to $L_{P-}$ structures and $L_{P}$-theories; and the notion of definitional equivalence itself: for structures $A+d_{\Phi} \equiv B+d_{\Theta}$; for theories, $T_{1}+d_{\Phi} \equiv T_{2}+d_{\Theta}$. Some results relating these notions were given, ending with two characterizations for definitional equivalence. In this second part, we explain the notion of a representation basis. Suppose a set $\Phi=\left\{\phi_{i}\right\}$ of $L_{P}$-formulas is given, and $\Theta=\left\{\theta_{i}\right\}$ is a set of $L_{Q}$-formulas. Then the original set $\Phi$ is called a representation basis for an $L_{P}$-structure $A$ with inverse $\Theta$ iff an inverse explicit definition $\forall \bar{x}\left(P_{i}(\bar{x}) \leftrightarrow \theta_{i}\right)$ is true in $A+d_{\Phi}$, for each $P_{i}$. Similarly, the set $\Phi$ is called a representation basis for a $L_{P}$-theory $T$ with inverse $\Theta$ iff each explicit definition $\forall \bar{x}\left(P_{i}(\bar{x}) \leftrightarrow \theta_{i}\right)$ is provable in $T+d_{\Phi}$. Some results about representation bases, the mappings they induce and their relationship with the notion of definitional equivalence are given. In particular, we show that $T_{1}$ (in $L_{P}$ ) is definitionally equivalent to $T_{2}$ (in $L_{Q}$ ), with respect to $\Phi$ and $\Theta$, if and only if $\Phi$ is a representation basis for $T_{1}$ with inverse $\Theta$ and $T_{2} \equiv D_{\Phi} T_{1}$.
\end{abstract}

Mathematics Subject Classification. Primary 03C07; Secondary 03C95.

Keywords. Definitional equivalence, Theories, Definability.

\section{Introduction}

Sometimes theories are formulated with different sets of non-logical primitives and yet are definitionally equivalent. There are many examples of theoriesoften involving formalized systems of arithmetic and set theory-formulated with rather different sets of primitives (aka signatures), which are nonetheless "equivalent". 


\section{Summary of Part I}

In Part I ([4]), we considered a starting language $L_{P}$ over a relational signature $P=\left\{P_{i}\right\}_{i \in I_{P}}$, and a set $\Phi=\left\{\phi_{i}\right\}_{i \in I}$ of $L_{P}$-formulas. Given $\Phi$, introduce a disjoint set $Q=\left\{Q_{i}\right\}_{i \in I}$ of new relation symbols, with card $Q=$ card $\Phi$, and with the arity of $Q_{i}$ matching the arity of $\phi_{i}$. The extended language is denoted $L_{P, Q}$ and the language built from the new signature $Q$ (with the implicitly induced arities) is denoted $L_{Q} \cdot{ }^{1}$

Definition 1. Given $\Phi=\left\{\phi_{i}\right\}$, the definition system over $\Phi$, which we write as,

$$
d_{\Phi}
$$

is the set of explicit definitions,

$$
\forall x_{1} \ldots x_{n_{i}}\left(Q_{i}\left(x_{1}, \ldots, x_{n_{i}}\right) \leftrightarrow \phi_{i}\right)
$$

where $\left\{x_{1}, \ldots x_{n_{i}}\right\}=\mathrm{FV}\left(\phi_{i}\right)$, and $n_{i}=$ card FV $\left(\phi_{i}\right)$ (the "arity" of $\phi_{i}$ ). These define the new symbols $Q_{i}$ in terms of the defining $L_{P}$-formulas $\phi_{i}$. We shall sometimes write $\forall \bar{x}\left(Q_{i}(\bar{x}) \leftrightarrow \phi_{i}\right)$ instead of $\forall x_{1} \ldots x_{n}\left(Q_{i}\left(x_{1}, \ldots, x_{n}\right) \leftrightarrow \phi_{i}\right){ }^{2}$

Definition 2. Let $A$ be an $L_{P}$-structure. Then $A+d_{\Phi}$ is the unique definitional expansion $A^{+}=d_{\Phi}$ of $A$. $\left(A+d_{\Phi}\right.$ is an $L_{P, Q}$-structure. $)$

Definition 3. Let $T$ be an $L_{P}$-theory. The the definitional extension of $T$ wrt $\Phi$ is $T+d_{\Phi}$. We say that $T^{+}$in $L_{P, Q}$ is a definitional extension of $T$ in $L_{P}$ just if

$$
T^{+} \equiv T+d_{\Phi},
$$

for some definition system $d_{\Phi}$, where $\Phi$ is some set of $L_{P}$-formulas.

Definition 4. Let a definition system $d_{\Phi}$ be given. Define the translation, induced by $\Phi$

$$
\tau_{\Phi}^{+}: L_{P, Q} \rightarrow L_{P}
$$

as follows. For symbols $P_{i}, Q_{j}$, variables $x, y, \bar{x}$, and for $L_{P, Q}$-formulas $\alpha, \alpha_{1}, \alpha_{2}$ :

$$
\begin{aligned}
\text { (1) } \tau_{\Phi}^{+}\left(P_{i}(\bar{x})\right) & :=P_{i}(\bar{x}) \\
\text { (2) } \tau_{\Phi}^{+}\left(Q_{j}(\bar{x})\right) & :=\left(\phi_{j}\right)^{\prime} \\
\text { (3) } \tau_{\Phi}^{+}(x=y) & :=(x=y) \\
\text { (4) } \tau_{\Phi}^{+}(\neg \alpha) & :=\neg \tau_{\Phi}^{+}(\alpha) \\
\text { (5) } \tau_{\Phi}^{+}\left(\alpha_{1} \# \alpha_{2}\right) & :=\tau_{\Phi}^{+}\left(\alpha_{1}\right) \# \tau_{\Phi}^{+}\left(\alpha_{2}\right) \\
\text { (6) } \tau_{\Phi}^{+}(\mathbf{q} x \alpha) & :=\mathbf{q} x \tau_{\Phi}^{+}(\alpha) .
\end{aligned}
$$

\footnotetext{
${ }^{1}$ To simplify notation, I sometimes write " $\Phi=\left\{\phi_{i}\right\}$ " to mean $\Phi=\left\{\phi_{i}\right\}_{i \in I}$, omitting the index set. In this case, the expression " $\left\{\phi_{i}\right\}$ " does not denote the singleton set containing $\phi_{i}$, but rather the indexed set $\left\{\phi_{i} \mid i \in I\right\}$ of such formulas.

${ }^{2}$ Strictly speaking, there is a distinct sequence $\bar{x}$ for each definition of a $Q_{i}$ symbol; but it would merely make notation ugly to keep mentioning that. Likewise, $d_{\Theta}$ will be an abbreviation for the set of definitions of the form $\forall \bar{x}\left(P_{i}(\bar{x}) \leftrightarrow \theta_{i}\right)$, where the $\theta_{i}$ are $L_{Q}$-formulas.
} 
where \# is any binary connective, $\mathbf{q}$ is a quantifier and $\left(\phi_{i}\right)^{\prime}$ is the result of ensuring that the free variables appearing $\phi_{i}$ are relabelled, to match those of $Q_{i}(\bar{x}) .{ }^{3}$ We call $\tau_{\Phi}^{+}$the translation induced by $\Phi$. It maps from the enriched language $L_{P, Q}$ back to the original language $L_{P}$. We let $\tau_{\Phi}$ be the restriction of $\tau_{\Phi}^{+}$to $L_{Q}$ : thus, $\tau_{\Phi}$ maps from the new language $L_{Q}$ back to the original language $L_{P}$. $\left(\tau_{\Phi}\right.$ is also called the translation induced by $\Phi$.)

Definition 5. Let $\tau_{\Phi}: L_{Q} \rightarrow L_{P}$ and $\tau_{\Theta}: L_{P} \rightarrow L_{Q}$ be translations induced by $d_{\Phi}$ and $d_{\Theta}$. Let $T_{1}$ be an $L_{P}$ theory. Let $T_{2}$ be an $L_{Q}$ theory. Then $\tau_{\Theta}$ is an right inverse of $\tau_{\Phi}$ in $T_{1}$ iff, for any $\alpha \in L_{P}$,

$$
T_{1} \vdash \alpha \leftrightarrow \tau_{\Phi}\left(\tau_{\Theta}(\alpha)\right)
$$

We write this more suggestively as:

$$
\left(\tau_{\Phi} \tau_{\Theta}=1\right)_{T_{1}}
$$

And $\tau_{\Theta}$ is an left inverse of $\tau_{\Phi}$ in $T_{2}$ iff, for any $\beta \in L_{Q}$,

$$
T_{2} \vdash \beta \leftrightarrow \tau_{\Theta}\left(\tau_{\Phi}(\beta)\right)
$$

Likewise, we write this more suggestively as:

$$
\left(\tau_{\Theta} \tau_{\Phi}=1\right)_{T_{2}}
$$

Definition 6. Let $A$ be an $L_{P}$-structure. Then the $L_{Q}$-structure $D_{\Phi} A$ is defined by:

$$
D_{\Phi} A:=\left.\left(A+d_{\Phi}\right)\right|_{L_{Q}}
$$

$D_{\Phi} A$ is called the definitional image of $A$ with respect to $\Phi$.

Definition 7. The definitional image of $T$, with respect to $\Phi$, is the restriction of the deductive closure of $T+d_{\Phi}$ to the new language $L_{Q}$. The definitional image of $T$ with respect to $\Phi$ is denoted $D_{\Phi} T$. That is,

$$
D_{\Phi} T:=\left.\operatorname{DedCl}\left(T+d_{\Phi}\right)\right|_{L_{Q}}=\left\{\beta \in L_{Q} \mid T+d_{\Phi} \vdash \beta\right\}
$$

Definition 8. Structures $A$ and $B$ are definitionally equivalent wrt $d_{\Phi}$ and $d_{\Theta}$ iff

$$
A+d_{\Phi} \cong B+d_{\Theta}
$$

If this is so, we write:

$$
A \underset{\Theta}{\stackrel{\Phi}{\longrightarrow}} B
$$

Definition 9. Theories $T_{1}$ and $T_{2}$ are definitionally equivalent wrt $d_{\Phi}$ and $d_{\Theta}$ iff

$$
T_{1}+d_{\Phi} \equiv T_{2}+d_{\Theta} .
$$

To express this, we write:

$$
T_{1} \underset{\Theta}{\stackrel{\Phi}{\leftrightarrow}} T_{2}
$$

In Part I we established a fair number of "book-keeping lemmas". The three most important results can be summarized:

\footnotetext{
${ }^{3}$ The clauses (4)-(6) are usually read as saying " $\tau_{\Phi}^{+}$commutes with the logical operators".
} 
Lemma 1. The following hold always: ${ }^{4}$

$$
\begin{aligned}
& \text { (1) If } A=T \text { then } D_{\Phi} A=D_{\Phi} T \\
& \text { (2) } D_{\Phi}[\operatorname{Mod}(T)] \subseteq \operatorname{Mod}\left(D_{\Phi} T\right)
\end{aligned}
$$

Lemma 2. The following are equivalent: ${ }^{5}$

$$
\begin{aligned}
& \text { (1) } T+d_{\Phi} \vdash d_{\Theta} \\
& \text { (2) }\left(\tau_{\Theta} \tau_{\Phi}=1\right)_{T} \\
& \text { (3) } T \underset{\Theta}{\stackrel{\Phi}{\longrightarrow}} D_{\Phi} T
\end{aligned}
$$

Lemma 3. The following are equivalent: ${ }^{6}$

$$
\begin{aligned}
& \text { (1) } A+d_{\Phi} \models d_{\Theta} \\
& \text { (2) } D_{\Theta} D_{\Phi} A=A \\
& \text { (3) } A \stackrel{\Phi}{\stackrel{\Theta}{\longrightarrow}} D_{\Phi} A
\end{aligned}
$$

In addition to those "book-keeping lemmas", we established two conditions for definitional equivalence, one for structures and one for theories:

Theorem 1. The following are equivalent:

$$
A \stackrel{\Phi}{\stackrel{\Theta}{\longrightarrow}} B
$$

(2) $B \cong D_{\Phi} A$ and $A \cong D_{\Theta} B$.

Theorem 2. The following are equivalent:

$$
T_{1} \stackrel{\Phi}{\stackrel{\Theta}{\longrightarrow}} T_{2}
$$

(2) $\left(\tau_{\Phi} \tau_{\Theta}=1\right)_{T_{1}}$ and $T_{2} \equiv D_{\Phi} T_{1}$.

\section{Definitional Equivalence: Model-Theoretic Criteria}

Theorem 2 above establishes a criterion for $T_{1} \underset{\Theta}{\stackrel{\Phi}{\longrightarrow}} T_{2}$ in terms of translation: $\left(\tau_{\Phi} \tau_{\Theta}=1\right)_{T_{1}}$ and $T_{2} \equiv D_{\Phi} T_{1}$. Next, we establish model-theoretic criteria.

Definition 10. We write

$$
\left(T_{1}, \Phi\right) \rightarrow\left(T_{2}, \Theta\right)
$$

to mean:

$$
\text { for any } A \models T_{1} \text {, there is a } B \models T_{2} \text { st } A+d_{\Phi} \cong B+d_{\Theta} \text {. }
$$

We write:

$$
\left(T_{1}, \Phi\right) \leftrightarrow\left(T_{2}, \Theta\right)
$$

to mean: $\left(T_{1}, \Phi\right) \rightarrow\left(T_{2}, \Theta\right)$ and $\left(T_{2}, \Theta\right) \rightarrow\left(T_{1}, \Phi\right)$.

\footnotetext{
${ }^{4}$ These correspond to Lemma 15(2) and 15(3), from Part I, [4].

${ }^{5}$ These correspond to Lemma 10(5), Lemma 11, Lemma 19 from Part I, [4].

${ }^{6}$ These are three conditions from Lemma 16 from Part I, [4].
} 
Lemma 4. If $\left(T_{1}, \Phi\right) \rightarrow\left(T_{2}, \Theta\right)$, then $D_{\Phi}: \operatorname{Mod}\left(T_{1}\right) \rightarrow \operatorname{Mod}\left(T_{2}\right)$ and, for any $A \in \operatorname{Mod}\left(T_{1}\right)$, we have $A \underset{\Theta}{\stackrel{\Phi}{\longrightarrow}} D_{\Phi} A$.

Proof. Let $\left(T_{1}, \Phi\right) \rightarrow\left(T_{2}, \Theta\right)$. So, for any $A \models T_{1}$, there is a $B \models T_{2}$ such that $A+d_{\Phi} \cong B+d_{\Theta}$.

Consider the operator $D_{\Phi}$. Let $A \models T_{1}$. So, there is a $B \models T_{2}$ such that $A+d_{\Phi} \cong B+d_{\Theta}$. So, $B \cong D_{\Phi} A$. So, $D_{\Phi} A=T_{2}$. So, $D_{\Phi}: \operatorname{Mod}\left(T_{1}\right) \rightarrow \operatorname{Mod}\left(T_{2}\right)$. And since $A+d_{\Phi} \cong B+d_{\Theta}$, we have $A+d_{\Phi} \cong D_{\Phi} A+d_{\Theta}$. So, $A \underset{\Theta}{\stackrel{\Phi}{\longrightarrow}} D_{\Phi} A$, as required.

Lemma 5. If $D_{\Phi}: \operatorname{Mod}\left(T_{1}\right) \rightarrow \operatorname{Mod}\left(T_{2}\right)$ and, for any $A \in \operatorname{Mod}\left(T_{1}\right)$, we have $A \stackrel{\Phi}{\leftrightarrow} D_{\Phi} A$, then $\left(T_{1}, \Phi\right) \rightarrow\left(T_{2}, \Theta\right)$.

Proof. Suppose that $D_{\Phi}: \operatorname{Mod}\left(T_{1}\right) \rightarrow \operatorname{Mod}\left(T_{2}\right)$ and, for any $A \in \operatorname{Mod}\left(T_{1}\right)$, we have $A \stackrel{\Phi}{\stackrel{\Theta}{\longrightarrow}} D_{\Phi} A$

Now suppose $A=T_{1}$. We claim there is a $B \models T_{2}$ st $A+d_{\Phi} \cong B+d_{\Theta}$.

Since $A \models T_{1}$, we have $D_{\Phi} A \models T_{2}$ and

$$
A \stackrel{\Phi}{\stackrel{\Theta}{\longrightarrow}} D_{\Phi} A
$$

And so,

$$
A+d_{\Phi} \cong D_{\Phi} A+d_{\Theta}
$$

So there is a $B \models T_{2}$ such that $A+d_{\Phi} \cong B+d_{\Theta}$. So, $\left(T_{1}, \Phi\right) \rightarrow\left(T_{2}, \Theta\right)$, as claimed.

Lemma 6. The following are equivalent:

$$
\left(T_{1}, \Phi\right) \rightarrow\left(T_{2}, \Theta\right) .
$$

(2) $D_{\Phi}: \operatorname{Mod}\left(T_{1}\right) \rightarrow \operatorname{Mod}\left(T_{2}\right)$ and, for any $A \in \operatorname{Mod}\left(T_{1}\right), A \underset{\Theta}{\stackrel{\Phi}{\longrightarrow}} D_{\Phi} A$.

Proof. Lemma 4 and Lemma 5.

Lemma 7. The following are equivalent:

$$
\begin{aligned}
& \text { (1) } T_{1}+d_{\Phi} \vdash T_{2}+d_{\Theta} . \\
& \text { (2) }\left(T_{1}, \Phi\right) \rightarrow\left(T_{2}, \Theta\right) .
\end{aligned}
$$

Proof. (1) $\Rightarrow(2)$. Assume $T_{1}+d_{\Phi} \vdash T_{2}+d_{\Theta}$. Recall Lemma 3, which implies:

(*) If $A+d_{\Phi} \models d_{\Theta}$, then $A+d_{\Phi} \cong D_{\Phi} A+d_{\Theta}$.

We wish to show $\left(T_{1}, \Phi\right) \rightarrow\left(T_{2}, \Theta\right)$. Let $A=T_{1}$. Thus, $A+d_{\Phi}=T_{1}+d_{\Phi}$. Thus, $A+d_{\Phi} \models T_{2}+d_{\Theta}$. And so, $A+d_{\Phi} \models d_{\Theta}$. From $(*)$, it follows that $A+d_{\Phi} \cong D_{\Phi} A+d_{\Theta}$. Since, $A+d_{\Phi} \models T_{2}$, we have $D_{\Phi} A+d_{\Theta} \models T_{2}+d_{\Theta}$. Thus, $D_{\Phi} A=T_{2}$. And $A+d_{\Phi} \cong D_{\Phi} A+d_{\Theta}$. I.e., $A \underset{\Theta}{\stackrel{\Phi}{\longrightarrow}} D_{\Phi} A$. Since this holds in general, $\left(T_{1}, \Phi\right) \rightarrow\left(T_{2}, \Theta\right)$.

$(2) \Rightarrow(1)$. We suppose, for any $A=T_{1}$, there is some $B \models T_{2}$ such that $A+d_{\Phi} \cong B+d_{\Theta}$. For a contradiction, suppose $T_{2}+d_{\Theta} \vdash \alpha$ and $T_{1}+d_{\Phi} \nvdash \alpha$, for some formula $\alpha$. This gives us $A+d_{\Phi} \models T_{1}+d_{\Phi}$, and $A+d_{\Phi} \not \models \alpha$. Thus, 
$A \models T_{1}$. Hence, there is a model $B \models T_{2}$ such that $A+d_{\Phi} \cong B+d_{\Theta}$. Thus, $B+d_{\Theta} \models T_{2}+d_{\Theta}$. Since $T_{2}+d_{\Theta} \vdash \alpha$, we have that $B+d_{\Theta} \models \alpha$. Since, $A+d_{\Phi} \cong B+d_{\Theta}$, we get $A+d_{\Phi}=\alpha$. Contradiction.

We then obtain a characterization theorem:

Theorem 3. The following are equivalent

$$
\begin{aligned}
& \text { (1) } T_{1} \stackrel{\Phi}{\Theta} T_{2} . \\
& \text { (2) }\left(T_{1}, \Phi\right) \leftrightarrow\left(T_{2}, \Theta\right) .
\end{aligned}
$$

Proof. Reason as follows:

$$
\begin{aligned}
T_{1} \stackrel{\Phi}{\Theta} T_{2} & \Leftrightarrow T_{1}+d_{\Phi} \equiv T_{2}+d_{\Theta} \\
& \Leftrightarrow T_{1}+d_{\Phi} \vdash T_{2}+d_{\Theta} \text { and } T_{2}+d_{\Theta} \vdash T_{1}+d_{\Phi} \\
& \Leftrightarrow\left(T_{1}, \Phi\right) \rightarrow\left(T_{2}, \Theta\right) \text { and }\left(T_{2}, \Theta\right) \rightarrow\left(T_{1}, \Phi\right) \\
& \Leftrightarrow\left(T_{1}, \Phi\right) \leftrightarrow\left(T_{2}, \Theta\right) .
\end{aligned}
$$

The relationship $\left(T_{1}, \Phi\right) \leftrightarrow\left(T_{2}, \Theta\right)$ asserts the existence of two functions $F: \operatorname{Mod}\left(T_{1}\right) \rightarrow \operatorname{Mod}\left(T_{2}\right)$ and $G: \operatorname{Mod}\left(T_{2}\right) \rightarrow \operatorname{Mod}\left(T_{1}\right)$ such that, for any $A \in \operatorname{Mod}\left(T_{1}\right), B \in \operatorname{Mod}\left(T_{2}\right)$, we have

$$
\begin{gathered}
F(A) \models T_{2} \text { and } A \stackrel{\Phi}{\stackrel{\Theta}{\longrightarrow}} F(A) \\
G(B) \mid=T_{1} \text { and } G(B) \stackrel{\Phi}{\stackrel{\Theta}{\longrightarrow}} B
\end{gathered}
$$

Together, these imply the existence of a bijection (wrt $\cong):^{7}$

$$
H: \operatorname{Mod}\left(T_{1}\right) \leftrightarrow \operatorname{Mod}\left(T_{2}\right)
$$

such that, for any $A \in \operatorname{Mod}\left(T_{1}\right)$, we have:

$$
A \underset{\Theta}{\stackrel{\Phi}{\longrightarrow}} H(A)
$$

To show this, first we prove a simple lemma about definitional equivalence of structures:

Lemma 8. If $A \underset{\Theta}{\stackrel{\Phi}{\longrightarrow}} B$ and $A^{\prime} \underset{\Theta}{\stackrel{\Phi}{\longrightarrow}} B$, then $A \cong A^{\prime}$.

Proof. Let $A+d_{\Phi} \cong B+d_{\Theta}$ and $A^{\prime}+d_{\Phi} \cong B+d_{\Theta}$. Thus, $A+d_{\Phi} \cong A^{\prime}+d_{\Phi}$. Thus, by right cancellation, $A \cong A^{\prime}$.

Lemma 9. If $\left(T_{1}, \Phi\right) \leftrightarrow\left(T_{2}, \Theta\right)$, there exists a bijection $H: \operatorname{Mod}\left(T_{1}\right) \leftrightarrow \operatorname{Mod}\left(T_{2}\right)$ wrt $\cong$ such that, for any $A \in \operatorname{Mod}\left(T_{1}\right), A \underset{\Theta}{\stackrel{\Phi}{\longrightarrow}} H(A)$.

\footnotetext{
${ }^{7}$ Whenever I use the notion of bijection relating classes of models, I mean "bijection up to isomorphism". We write $H: \operatorname{Mod}\left(T_{1}\right) \leftrightarrow \operatorname{Mod}\left(T_{2}\right)$ to mean the map $H: \operatorname{Mod}\left(T_{1}\right) \rightarrow$ $\operatorname{Mod}\left(T_{2}\right)$ is a bijection.
} 
Proof. The assumption $\left(T_{1}, \Phi\right) \leftrightarrow\left(T_{2}, \Theta\right)$ asserts the existence of two functions $F, G$ which are linked by the parameters $\Phi, \Theta$. We show that $G$ left-inverts $F$ and $F$ left-inverts $G$.

Let $A \in \operatorname{Mod}\left(T_{1}\right)$. Then $F(A) \in \operatorname{Mod}\left(T_{2}\right)$ and

$$
A \stackrel{\Phi}{\stackrel{\Phi}{\longrightarrow}} F(A) \text {. }
$$

Now consider $G(F(A))$. We have $G(F(A)) \in \operatorname{Mod}\left(T_{1}\right)$ and

$$
G(F(A)) \stackrel{\Phi}{\stackrel{\Theta}{\longrightarrow}} F(A) .
$$

So, by the above cancellation lemma, $G(F(A)) \cong A$. Thus $G$ is a left-inverse of $F$.

We may also show that $F$ is a right inverse of $G$. For suppose $B \in$ $\operatorname{Mod}\left(T_{2}\right)$. Then $G(B) \in \operatorname{Mod}\left(T_{1}\right)$ and

$$
B \underset{\Phi}{\stackrel{\Theta}{\longrightarrow}} G(B) \text {. }
$$

Likewise, consider $F(G(B))$. We have $F(G(B)) \in \operatorname{Mod}\left(T_{2}\right)$ and

$$
F(G(B)) \stackrel{\Theta}{\stackrel{\Theta}{\longrightarrow}} G(B) .
$$

So, by the above cancellation lemma again, $F(G(B)) \cong B$. Thus $F$ is a leftinverse of $G$.

Now if we have $F: X \rightarrow Y$ and $G: Y \rightarrow X$, and $G$ is a left-inverse of $F$ and $F$ is a left-inverse of $G$, then $F: X \rightarrow Y$ is a bijection. Therefore, $F: \operatorname{Mod}\left(T_{1}\right) \rightarrow \operatorname{Mod}\left(T_{2}\right)$ satisfies the conditions stated.

Lemma 10. If $\left(T_{1}, \Phi\right) \leftrightarrow\left(T_{2}, \Theta\right)$, then $D_{\Phi}: \operatorname{Mod}\left(T_{1}\right) \rightarrow \operatorname{Mod}\left(T_{2}\right)$ is a bijection wrt $\cong$ such that, for any $A \in \operatorname{Mod}\left(T_{1}\right), A \underset{\Theta}{\stackrel{\Phi}{\longrightarrow}} D_{\Phi}(A)$.

Proof. Suppose $\left(T_{1}, \Phi\right) \leftrightarrow\left(T_{2}, \Theta\right)$. So, by Lemma 9 , there exists a bijection $H: \operatorname{Mod}\left(T_{1}\right) \leftrightarrow \operatorname{Mod}\left(T_{2}\right)$ wrt $\cong$ such that, for any $A \in \operatorname{Mod}\left(T_{1}\right), A \underset{\Theta}{\stackrel{\Phi}{\longrightarrow}} H(A)$. Thus, for any $A \in \operatorname{Mod}\left(T_{1}\right)$, we have,

$$
A+d_{\Phi} \cong H(A)+d_{\Theta}
$$

We will show that $H$ and $D_{\Phi}$ are the same up to isomorphism: i.e.,

$$
H(A) \cong D_{\Phi} A
$$

Since $\left(T_{1}, \Phi\right) \rightarrow\left(T_{2}, \Theta\right)$, by Lemma 4 , we know that $D_{\Phi}: \operatorname{Mod}\left(T_{1}\right) \rightarrow \operatorname{Mod}\left(T_{2}\right)$ and, for any $A \in \operatorname{Mod}\left(T_{1}\right)$, we have $A \underset{\Theta}{\stackrel{\Phi}{\longrightarrow}} D_{\Phi} A$. So, for any $A \in \operatorname{Mod}\left(T_{1}\right)$, we have

(ii) $\quad A+d_{\Phi} \cong D_{\Phi} A+d_{\Theta}$

Combining (i) and (ii), we infer,

$$
\text { (iii) } H(A)+d_{\Theta} \cong D_{\Phi} A+d_{\Theta}
$$

And by cancellation, $H(A) \cong D_{\Phi} A$, as required.

Furthermore, the converse is true. 
Lemma 11. If $D_{\Phi}: \operatorname{Mod}\left(T_{1}\right) \leftrightarrow \operatorname{Mod}\left(T_{2}\right)$ such that, for any $A \in \operatorname{Mod}\left(T_{1}\right)$, $A \underset{\Theta}{\stackrel{\Phi}{\longrightarrow}} D_{\Phi} A$, then $\left(T_{1}, \Phi\right) \leftrightarrow\left(T_{2}, \Theta\right)$.

Proof. Let $D_{\Phi}: \operatorname{Mod}\left(T_{1}\right) \rightarrow \operatorname{Mod}\left(T_{2}\right)$ be a bijection such that, for any $A \in$ $\operatorname{Mod}\left(T_{1}\right)$

$$
A+d_{\Phi} \cong D_{\Phi} A+d_{\Theta} .
$$

We want to show, first, that for, any $A \models T_{1}$, there is a $B \models T_{2}$ st $A+d_{\Phi} \cong$ $B+d_{\Theta}$. If $A=T_{1}$, then $D_{\Phi} A$ is such a model. So, $\left(T_{1}, \Phi\right) \rightarrow\left(T_{2}, \Theta\right)$. We want to show, second, that for, any $B \models T_{2}$, there is an $A=T_{1}$ st $A+d_{\Phi} \cong B+d_{\Theta}$. If $B \models T_{2}$, then $D_{\Phi}^{-1} B$ is such a model. So, $\left(T_{2}, \Theta\right) \rightarrow\left(T_{1}, \Phi\right)$. And therefore we have $\left(T_{1}, \Phi\right) \leftrightarrow\left(T_{2}, \Theta\right)$.

The two previous lemmas give a second characterization theorem:

Theorem 4. The following are equivalent:

$$
\begin{aligned}
& \left(T_{1}, \Phi\right) \leftrightarrow\left(T_{2}, \Theta\right) . \\
& D_{\Phi}: \operatorname{Mod}\left(T_{1}\right) \leftrightarrow \operatorname{Mod}\left(T_{2}\right) \text { st for any } A \in \operatorname{Mod}\left(T_{1}\right), A \underset{\Theta}{\stackrel{\Phi}{\longleftrightarrow}} D_{\Phi} A .
\end{aligned}
$$

\section{Mutual Definability Does Not Imply Definitional Equivalence}

Andréka et al ([1]) show that mutual definability of a pair of theories in each other does not entail their definitional equivalence. First, their notion of modeltheoretic definability is explained as follows:

Let $T h 1$ and Th2 be theories, maybe on different first-order languages. An explicit definition of $T h 1$ over $T h 2$ is a conjunction $\Delta$ of explicit definitions of the relation symbols of $T h 1$ in terms of the language of $T h 2$ such that the models of $T h 1$ are exactly the reducts of the models of $T h 2 \cup \Delta$ (to the language of $T h 1$ ). Thus, we get the models of $T h 1$ from those of $T h 2$ by first defining the relations of Th1 via using $\Delta$, and then forgetting the relations not present in the language of $T h 2$. ([1]: 591)

If we switch their $T h 1$ to $T_{2}, T h 2$ to $T_{1}$, and $\Delta$ to $d_{\Phi}$, this corresponds, in our terminology, to saying that $\Phi$ model-theoretically defines $T_{2}$ in $T_{1}$ : i.e., $\operatorname{Mod}\left(T_{2}\right)=D_{\Phi}\left[\operatorname{Mod}\left(T_{1}\right)\right]($ see [4], Definition 28, Part I).

It is relatively straightforward to prove:

Lemma 12. Let $T_{1} \underset{\Theta}{\stackrel{\Phi}{\longrightarrow}} T_{2}$. Then

$$
\begin{aligned}
& \text { (1) } \operatorname{Mod}\left(T_{2}\right)=D_{\Phi}\left[\operatorname{Mod}\left(T_{1}\right)\right] \\
& \text { (2) } \operatorname{Mod}\left(T_{1}\right)=\operatorname{D}_{\Theta}\left[\operatorname{Mod}\left(T_{2}\right)\right] .
\end{aligned}
$$

Proof. Let us suppose that $T_{1} \underset{\Theta}{\stackrel{\Phi}{\hookrightarrow}} T_{2}$. That is, $T_{1}+d_{\Phi} \equiv T_{2}+d_{\Theta}$.

We first wish to prove $(1): \operatorname{Mod}\left(T_{2}\right)=D_{\Phi}\left[\operatorname{Mod}\left(T_{1}\right)\right]$. That is, we wish to prove that, for any $L_{Q}$-structure $B$, we have:

$$
B \models T_{2} \text { if and only if } B \cong D_{\Phi} A \text {, for some } A \models T_{1} \text {. }
$$


Suppose $B \models T_{2}$. Thus, $B+d_{\Theta} \models T_{2}+d_{\Theta}$. So, $B+d_{\Theta} \models T_{1}+d_{\Phi}$. Let $A=D_{\Theta} B$. Now $B+d_{\Theta} \models d_{\Phi}$. So, from Lemma 3 (but relabelling in terms of

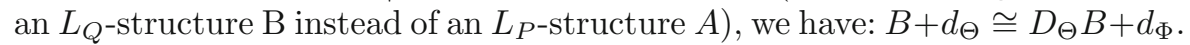
I.e., $B+d_{\Theta} \cong A+d_{\Phi}$. So, $A+d_{\Phi} \models T_{1}+d_{\Phi}$. So, $A \models T_{1}$, as required.

Instead, suppose $B \cong D_{\Phi} A$, where $A \models T_{1}$. Thus, $A+d_{\Phi} \models T_{1}+d_{\Phi}$. And thus, $A+d_{\Phi} \models T_{2}+d_{\Theta}$. Since $A+d_{\Phi} \models d_{\Theta}$, we have, by Lemma 3 again, $A+d_{\Phi} \cong D_{\Theta} A+d_{\Theta}$. Thus, $A+d_{\Phi} \cong B+d_{\Theta}$. And so, $B+d_{\Theta}=T_{2}+d_{\Theta}$, which implies that $B \models T_{2}$, as required.

The proof of $(2), \operatorname{Mod}\left(T_{1}\right)=D_{\Theta}\left[\operatorname{Mod}\left(T_{2}\right)\right]$, is entirely analogous, just switching labels.

On the other hand, the converse of Lemma 12 is not true. Andréka et al 2005 [1] provide a counter-example:

Theorem 5. (Andréka et al 2005 [1]) There are theories $T_{1}, T_{2}$ and defining sets $\Phi, \Theta$ such that the following all hold:

(1) $\operatorname{Mod}\left(T_{2}\right)=D_{\Phi}\left[\operatorname{Mod}\left(T_{1}\right)\right]$.

(2) $\operatorname{Mod}\left(T_{1}\right)=D_{\Theta}\left[\operatorname{Mod}\left(T_{2}\right)\right]$.

(3) $\operatorname{not}-\left(T_{1} \underset{\Theta^{\prime}}{\stackrel{\Phi^{\prime}}{\longrightarrow}} T_{2}\right.$, for any $\left.\Phi^{\prime}, \Theta^{\prime}\right)$.

One way to understand the problem is that the translations associated with $\Phi$ and $\Theta$ are not mutual inverses. Below (Theorem 16) we show the "gap" connected to Lemma 12 and Theorem 5 closes, by requiring not only $\operatorname{Mod}\left(T_{2}\right)=D_{\Phi}\left[\operatorname{Mod}\left(T_{1}\right)\right]$ but also that $\Phi$ is a representation basis for $T_{1}$ with inverse $\Theta$ : if these conditions hold, then it follows that $T_{1} \underset{\Theta}{\stackrel{\Phi}{\longrightarrow}} T_{2} \cdot{ }^{8}$

\section{Summary}

We quickly summarize the results of the three previous sections.

Theorem 6. The following three claims are equivalent:

(2) $D_{\Phi}: \operatorname{Mod}\left(T_{1}\right) \rightarrow \operatorname{Mod}\left(T_{2}\right)$ st, for any $A \in \operatorname{Mod}\left(T_{1}\right), A \underset{\Theta}{\stackrel{\Phi}{\longrightarrow}} D_{\Phi} A . \quad(L e m m a 6)$

$$
\left.\left(T_{1}, \Phi\right) \rightarrow\left(T_{2}, \Theta\right) \text {. (Lemma } 7\right)
$$

Theorem 7. The following are equivalent:

(2) $\quad A+d_{\Phi} \cong B+d_{\Theta}$. (Definition 8)

(3) $B \cong D_{\Phi} A$ and $A \cong D_{\Theta} B$. (Theorem 1 )

\footnotetext{
${ }^{8}$ Moreover, the condition " $\Phi$ is a representation basis for $T_{1}$ with inverse $\Theta$ " is equivalent to the mutual invertibility condition of translations, that $\left(\tau_{\Phi} \tau_{\Theta}=1\right)_{T_{1}}$. See Theorem 10 below (it follows immediately from Lemma 1).
} 
Theorem 8. The following are equivalent: ${ }^{9}$

$$
\begin{gathered}
T_{1} \stackrel{\Phi}{\Theta} T_{2} . \\
T_{1}+d_{\Phi} \equiv T_{2}+d_{\Theta} .(\text { Definition } 9) \\
\left(\tau_{\Theta} \tau_{\Phi}=1\right)_{T_{1}} \text { and } T_{2} \equiv D_{\Phi} T_{1} .(\text { Theorem } 2) \\
\left(T_{1}, \Phi\right) \leftrightarrow\left(T_{2}, \Theta\right) .(\text { Theorem } 3)
\end{gathered}
$$

(5) $D_{\Phi}: \operatorname{Mod}\left(T_{1}\right) \leftrightarrow \operatorname{Mod}\left(T_{2}\right)$ st, for any $A \in \operatorname{Mod}\left(T_{1}\right), A \underset{\Theta}{\stackrel{\Phi}{\hookrightarrow}} D_{\Phi} A$. (Theorem 4)

\section{Representation Basis}

We next move on to defining the notion of "representation basis" for a structure and for a theory.

The underlying intuitive concept is fairly simple. Given a structure $A$ in a signature $P$, we may wish to consider a special set $\Phi$ of $L_{P}$-formulas, and then examine the "internal structure" defined by them in $A$ : this is what we have called the "definitional image", $D_{\Phi} A$. What condition should we impose if we wish to reconstruct $A$ from its image $D_{\Phi} A$ ?

Clearly, the condition is that the definitional expansion $A+d_{\Phi}$-which we used to define $D_{\Phi} A$ prior to forgetting the $P$-relations - should satisfy an invertibility condition, namely that each original $P_{i}$ be explicitly definable from a formula, say $\theta_{i}$, in the new $Q$-language. This then means that the original set $\Phi$ of $L_{P}$-formulas, in some sense, does not "omit" any structural content built into $A$ itself. The formulas $\phi_{i}$ merely "encode" that content differently.

That condition is then, simply, that there is a set $\Theta=\left\{\theta_{i}\right\}_{i \in I_{P}}$ of $Q$ formulas such that

$$
A+d_{\Phi} \models d_{\Theta}
$$

holds. Or, more explicitly, for each atomic formula $P_{i}(\bar{x})$ of $L_{P}$, we have an explicit inverse definition,

$$
A+d_{\Phi}=\forall \bar{x}\left(P_{i}(\bar{x}) \leftrightarrow \theta_{i}\right)
$$

for some $\theta_{i} \in L_{Q}$.

The same intuition motivates an analogous account for theories. The definitional image $D_{\Phi} T$ is a smaller theory which "lives inside" the original $T$ (though it is not a sub-theory). It is kind of filtering or projection. But what condition would permit reconstruction of the original?

Again, it is the condition that there is a set $\Theta=\left\{\theta_{i}\right\}_{i \in I_{P}}$ of $Q$-formulas such that

$$
T+d_{\Phi} \vdash d_{\Theta}
$$

\footnotetext{
${ }^{9}$ Aside from the analysis of definitional equivalence in terms of "concept algebras", these are similar to the four characterizations recently given in the lecture notes Andréka \& Németi 2014 ([2], p. 40), except that my second condition (Theorem 2) seems to be much stronger than theirs.
} 
holds. Similarly, we can more explicitly express this by saying that, for each atomic formula $P_{i}(\bar{x})$ of $L_{P}$, we have an explicit inverse definition,

$$
T+d_{\Phi} \vdash \forall \bar{x}\left(P_{i}(\bar{x}) \leftrightarrow \theta_{i}\right)
$$

for some $\theta_{i} \in L_{Q}$.

As the reader may have noticed above, throughout earlier sections, we have examined what kinds of consequences follow from precisely this invertibility assumption. Based on these informal explanations, we then given the two main definitions:

Definition 11. $\Phi$ is a representation basis for $A$ with inverse $\Theta$ iff $A+d_{\Phi}=d_{\Theta}$.

Definition 12. $\Phi$ is a representation basis for $T$ with inverse $\Theta$ iff $T+d_{\Phi} \vdash d_{\Theta}$.

In each case, the formulas $\theta_{i}$ in the set $\Theta$ are called inversion formulas. The following two lemmas are entirely straightforward.

Lemma 13. If $T$ is inconsistent, any $\Phi$ is a representation basis for $T$.

Lemma 14. Suppose $T+d_{\Phi} \vdash d_{\Theta}$ and $T+d_{\Phi} \vdash d_{\Psi}$, for inverses $\Theta=\left\{\theta_{i}\right\}$ and $\Psi=\left\{\psi_{i}\right\}$. Then, for all $i$, we have: $T+d_{\Phi} \vdash \theta_{i} \leftrightarrow \psi_{i}$.

For the case where $T$ is the empty theory in $L_{P}$ (i.e., pure logic), we define the notion of a logical representation basis (for $L_{P}$ ):

Definition 13. $\Phi$ is a logical representation basis for $L_{P}$ with inverse $\Theta$ iff $d_{\Phi} \vdash d_{\Theta}$.

It is easy to see that being a representation basis is preserved under theory extension (or structure expansion):

Lemma 15. If $\Phi$ is a representation basis for $T$ (or structure $A$ ), then $\Phi$ is also a representation basis for every extension of $T$ (resp. every expansion of $A)$. In particular, if $\Phi$ is a logical representation basis (for $L_{P}$ ), then it is a representation basis for every theory $T$ (in $L_{P}$ ).

The converse of this lemma is not true. $\Phi$ might be a representation basis for $T$, but not a representation basis for a sub-theory or a weaker theory. Similarly, $\Phi$ might be a representation basis for $A$, but not a representation basis for a reduct of $A$.

We note in passing that while we have defined representation basis syntactically in terms of explicit definability of the $P_{i}$, a mathematically equivalent model-theoretic definition can be given, in terms of implicit definability:

Definition 14. A set $\Phi$ of $L_{P}$-formulas is a semantic representation basis for $T$ just in case, each primitive $L_{P}$-symbol $P_{i}$ is implicitly definable in $T+d_{\Phi}$.

It then follows, using Beth's definability theorem, that the syntactic definition of representation basis (Definition 12) and the model-theoretic one (Definition 14) are equivalent:

Theorem 9. $\Phi$ is a representation basis for $T$ iff $\Phi$ is a semantic representation basis for $T$. 


\section{Examples}

We give three examples of this notion in operation and an interesting example involving definitional image. The first three are fairly simple. The fourth provides an example of a logical representation basis $\Phi$ for (a propositional language) $L_{P}$ but where the definitional image of logic in $L_{P}$ under $D_{\Phi}$ is not logically true.

Example 1. Given a signature $P=\left\{P_{i}\right\}_{i \in I_{P}}$, let $\Phi=\left\{P_{i}\left(x_{1}, \ldots, x_{a\left(P_{i}\right)}\right)\right\}_{i \in I_{P}}$, consisting of the atomic formulas of the language $L_{P}$. Then $\Phi$ is a logical representation basis for $L_{P}$, and indeed a representation basis for any theory $T$ in $L_{P}$. In this case, each new $Q_{i}$ is simply defined as $P_{i}$. This means they are equivalent in the extension $T+d_{\Phi}$. And then, trivially, the inversion conditions hold.

The next two examples are slightly modified from examples given by David Miller in his series of papers explaining the language-dependence problem for explications of the concept of truthlikeness (Miller 1974 [5], 1975 [6], $1978[7])$.

Example 2. Let $P=\left\{p_{1}, p_{2}\right\}$ be a propositional signature and consider the $L_{P}$-formulas

$$
\begin{aligned}
\phi_{1} & :=p_{1} \\
\phi_{2} & :=p_{1} \leftrightarrow p_{2}
\end{aligned}
$$

Then $\left\{\phi_{1}, \phi_{2}\right\}$ is a logical representation basis for $L_{P}$. For consider the definitions of the new symbols, $Q_{1}$ and $Q_{2}$ :

$$
\begin{aligned}
Q_{1} & \leftrightarrow p_{1} \\
Q_{2} & \leftrightarrow\left(p_{1} \leftrightarrow p_{2}\right)
\end{aligned}
$$

Let $d_{\Phi}$ be $\left\{Q_{1} \leftrightarrow p_{1}, Q_{2} \leftrightarrow\left(p_{1} \leftrightarrow p_{2}\right)\right\}$. Then $d_{\Phi}$ implies:

$$
\begin{aligned}
& p_{1} \leftrightarrow Q_{1} \\
& p_{2} \leftrightarrow\left(Q_{1} \leftrightarrow Q_{2}\right)
\end{aligned}
$$

So, given $d_{\Phi}, p_{1}$ and $p_{2}$ can be explicitly defined in terms of $Q_{1}$ and $Q_{2}$ (this happens essentially because $p_{1} \leftrightarrow\left(p_{1} \leftrightarrow p_{2}\right)$ is logically equivalent to $\left.p_{2}\right)$.

Example 3. Let $P=\left\{P_{1}, P_{2}\right\}$, where $P_{1}, P_{2}$ are unary predicates. Consider the $L_{P}$-formulas $\phi_{1}, \phi_{2}$ :

$$
\begin{aligned}
& \phi_{1}:=P_{1}(x) \\
& \phi_{2}:=P_{1}(x) \leftrightarrow P_{2}(x)
\end{aligned}
$$

Then the pair $\left\{\phi_{1}, \phi_{2}\right\}$ is a logical representation basis for $L_{P}$. For the explicit definitions,

$$
\begin{aligned}
& Q_{1}(x) \leftrightarrow P_{1}(x) \\
& Q_{2}(x) \leftrightarrow\left(P_{1}(x) \leftrightarrow P_{2}(x)\right)
\end{aligned}
$$


imply (in logic alone) the inversions:

$$
\begin{aligned}
& P_{1}(x) \leftrightarrow Q_{1}(x) \\
& P_{2}(x) \leftrightarrow\left(Q_{1}(x) \leftrightarrow Q_{2}(x)\right)
\end{aligned}
$$

for the same reason as the previous example.

Example 4. Consider, given $\Phi$, the definitional image of $T$ when $T$ is the empty theory. Let $\log _{P}$ be the set of $L_{P}$-sentences which are theorems of logic in $L_{P}$. The definitional image $\log _{P}$ under $\Phi$ is given by:

$$
D_{\Phi} \log _{P}:=\left\{\beta \in \operatorname{Sent}\left(L_{Q}\right) \mid d_{\Phi} \vdash \beta\right\}
$$

Then:

Observation. There is a signature $P$ and (logical) representation basis $\Phi$ for logic in $L_{P}$ such that the $L_{Q}$ theory $D_{\Phi} \log _{P}$ isn't logically true.

For example, let $P=\left\{p_{1}, p_{2}\right\}$ be a propositional signature and consider the three $L_{P}$-formulas:

$$
\begin{aligned}
\phi_{1} & :=p_{1} \wedge p_{2} \\
\phi_{2} & :=p_{1} \wedge \neg p_{2} \\
\phi_{3} & :=\neg p_{1} \wedge p_{2}
\end{aligned}
$$

Then one can show that $\left\{\phi_{1}, \phi_{2}, \phi_{3}\right\}$ is a logical representation basis for $L_{P}$. Introduce the definition system $d_{\Phi}$ for the new symbols, $Q_{1}, Q_{2}$ and $Q_{3}$ :

$$
\begin{aligned}
& Q_{1} \leftrightarrow p_{1} \wedge p_{2} \\
& Q_{2} \leftrightarrow p_{1} \wedge \neg p_{2} \\
& Q_{3} \leftrightarrow \neg p_{1} \wedge p_{2}
\end{aligned}
$$

Then inversions - i.e., explicit definitions of $p_{1}, p_{2}$ in terms of the $Q_{i}$ - can be obtained as follows:

$$
\begin{aligned}
& d_{\Phi} \vdash p_{1} \leftrightarrow\left(Q_{1} \vee Q_{2}\right) \\
& d_{\Phi} \vdash p_{2} \leftrightarrow\left(Q_{1} \vee Q_{3}\right)
\end{aligned}
$$

However, not every $L_{Q}$-theorem of $d_{\Phi}$ is logically true. For example,

$$
\begin{aligned}
& d_{\Phi} \vdash Q_{1} \rightarrow \neg Q_{2} \\
& d_{\Phi} \vdash Q_{1} \rightarrow \neg Q_{3} \\
& d_{\Phi} \vdash Q_{2} \rightarrow \neg Q_{3}
\end{aligned}
$$

In each case, we have $\beta \in L_{Q}$, with $d_{\Phi} \vdash \beta$. But, for each $\beta$, we have $\nvdash \beta$. ${ }^{10}$ And thus the theory $D_{\Phi} \log _{P}$ is not logically true.

${ }^{10}$ Note that $\vdash \tau_{\Phi}(\beta)$. 


\section{Basis, Translation and Equivalence}

We can now begin assemble the various pieces of this rather complicated jigsaw. In Subsection 8.1, we shall establish equivalent ways of expressing " $\Phi$ is a representation basis for $T$ with inverse $\Theta$ ". Then, in Subsection 8.2, we shall see how imposing this condition leads to strengthened properties of the $D_{\Phi}$ operator. Finally, in Subsection 8.3, we see how to include being a representation basis as a further criterion for expressing " $T_{1}$ is definitionally equivalent to $T_{2}$, wrt $\Phi$ and $\Theta "$.

\subsection{Criteria for Being a Representation Basis}

First, we establish equivalents for $\Phi$ being a representation basis for $T$ with inverse $\Theta$.

Theorem 10. The following conditions are equivalent:

(1) $\Phi$ is a representation basis for $T$ with inverse $\Theta$.

$$
\begin{aligned}
& T+d_{\Phi} \vdash d_{\Theta} . \\
& \left(\tau_{\Phi} \tau_{\Theta}=1\right)_{T} . \\
& T \stackrel{\Phi}{\stackrel{\leftrightarrow}{\longrightarrow}} D_{\Phi} T .
\end{aligned}
$$

For any $A \models T, A \underset{\Theta}{\stackrel{\Phi}{\longrightarrow}} D_{\Phi} A$.

For any $A=T, D_{\Theta} D_{\Phi} A=A$.

Proof. (1) $\Leftrightarrow(2)$ is simply Definition 12 . (2) $\Leftrightarrow$ (3) follows immediately from Lemma 2. Similarly, (2) $\Leftrightarrow$ (4) follows immediately from Lemma 2 .

For $(2) \Leftrightarrow(5)$ : first, suppose $T+d_{\Phi} \vdash d_{\Theta}$. So, by Lemma $2: T+d_{\Phi} \equiv$ $D_{\Phi} T+d_{\Theta}$. Next, suppose $A \models T$. So, $A+d_{\Phi} \models d_{\Theta}$. So, by Lemma 3 : $A+d_{\Phi} \cong$ $D_{\Phi} A+d_{\Theta}$. So, $A \underset{\Theta}{\stackrel{\Phi}{\longrightarrow}} D_{\Phi} A$, as claimed.

For the converse, suppose $A \stackrel{\Phi}{\stackrel{\Phi}{\longrightarrow}} D_{\Phi} A$, for any $A=T$. By Lemma 3, we infer that $A+d_{\Phi} \models d_{\Theta}$, for any $A \models T$. Now suppose $B \models T+d_{\Phi}$. Let $B=A^{\prime}+d_{\Phi}$. So, $A^{\prime} \models T$. And therefore, $A^{\prime}+d_{\Phi} \models d_{\Theta}$. So, $B \models d_{\Theta}$. Therefore, $T+d_{\Phi} \vdash d_{\Theta}$, as claimed.

For $(5) \Leftrightarrow(6)$ : notice that, by Lemma 3,

$$
D_{\Theta} D_{\Phi} A=A \text { iff } A \stackrel{\Phi}{\stackrel{\Phi}{\longrightarrow}} D_{\Phi} A
$$

Next, specializing to the case where $T$ is pure logic, we obtain: 
Theorem 11. The following are equivalent:

(1) $\Phi$ is a logical representation basis for $L_{P}$ with inverse $\Theta$.

$$
\begin{gathered}
d_{\Phi} \vdash d_{\Theta} . \\
\vdash \alpha \leftrightarrow \tau_{\Phi}\left(\tau_{\Theta}(\alpha)\right), \quad \text { for any } \alpha \in L_{P} . \\
A+d_{\Phi} \models d_{\Theta}, \quad \text { for any } A . \\
A \stackrel{\Phi}{\rightleftarrows} D_{\Phi} A, \quad \text { for any } A . \\
D_{\Theta} D_{\Phi} A \cong A, \quad \text { for any } A .
\end{gathered}
$$

Proof. The equivalences of (1), (2) and (3) follow immediately from Theorem 10 by setting $T$ as the empty theory. The equivalences of (4), (5) and (6) follow immediately from Lemma 3. And the equivalence of (2) and (5) follows from the equivalence of conditions (2) and (5) in Theorem 10, by setting $T$ as the empty theory.

\subsection{Consequences of $\Phi$ Being a Representation Basis for $\boldsymbol{T}$}

Theorem 12. Let $\Phi$ be a representation basis for $T$ with inverse $\Theta$. Then:

$$
D_{\Phi}: \operatorname{Mod}(T) \leftrightarrow \operatorname{Mod}\left(D_{\Phi} T\right) .
$$

(2) If $A, B \in \operatorname{Mod}(T)$ and $D_{\Phi} A \cong D_{\Phi} B$, then $A \cong B$.

(3) For any $B=D_{\Phi} T$, there is $A=T$ st $B \cong D_{\Phi} A$.

$$
\begin{gathered}
A=T \text { iff } D_{\Phi} A \models D_{\Phi} T . \\
D_{\Phi}[\operatorname{Mod}(T)]=\operatorname{Mod}\left(D_{\Phi} T\right) .
\end{gathered}
$$

Proof. Let us suppose $\Phi$ is a representation basis for $T$ with inverse $\Theta$. In particular, by Theorem 10(4), we have:

$$
\text { (*) } T \stackrel{\Phi}{\stackrel{\Theta}{\longrightarrow}} D_{\Phi} T
$$

Second, recall Theorem 8. Conditions (1) and (5) tell us that $T_{1} \underset{\Theta}{\stackrel{\Phi}{\longrightarrow}} T_{2}$ iff $D_{\Phi}: \operatorname{Mod}\left(T_{1}\right) \leftrightarrow \operatorname{Mod}\left(T_{2}\right)$ st, for any $A \in \operatorname{Mod}\left(T_{1}\right), A \underset{\Theta}{\stackrel{\Phi}{\longrightarrow}} D_{\Phi} A$. So, we have, using $(*)$ :

$$
\text { (**) } \quad D_{\Phi}: \operatorname{Mod}(T) \leftrightarrow \operatorname{Mod}\left(D_{\Phi} T\right) \text { st, for any } A \in \operatorname{Mod}(T), A \stackrel{\Phi}{\hookrightarrow} D_{\Phi} A .
$$

We can then prove the results quickly. of (1).

(1) is a consequence of (**). And (2) and (3) are obvious consequences

For (4). We already know that if $A \models T$, then $D_{\Phi} A=D_{\Phi} T$ (Lemma 1(1)). So, instead, suppose that $D_{\Phi} A \models D_{\Phi} T$. Let $B=D_{\Phi} A$. It follows from (**) that there is a model $A^{\prime}=T$ such that $B \cong D_{\Phi} A^{\prime}$. So $B \cong D_{\Phi} A^{\prime}$ and $B \cong D_{\Phi} A$, which implies: $D_{\Phi} A^{\prime} \cong D_{\Phi} A$. And therefore, $A \cong A^{\prime}$, since $D_{\Phi}$ is injective. So, $A \models T$.

For $(5)$. We already know that $D_{\Phi}[\operatorname{Mod}(T)] \subseteq \operatorname{Mod}\left(D_{\Phi} T\right)(\operatorname{Lemma} 1(2))$. We wish to prove the converse: $\operatorname{Mod}\left(D_{\Phi} T\right) \subseteq D_{\Phi}[\operatorname{Mod}(T)]$. So let $B \in \operatorname{Mod}\left(D_{\Phi} T\right)$. 
We claim $B \in D_{\Phi}[\operatorname{Mod}(T)]$. That is, we claim there is some $A \models T$ such that $B \cong D_{\Phi} A$. But this follows immediately from (3).

The next two results reveal the sense in which moving between different bases, say $\Phi=\left\{\phi_{i}\right\}$ and $\Phi^{*}=\left\{\phi_{i}^{*}\right\}$, is somewhat analogous to moving between bases for a vector space or between co-ordinate system on a manifold. That is, so long as both are representation bases, then they are interdefinable:

Theorem 13. Let an $L_{P}$-structure $A$ be given. Let $\Phi=\left\{\phi_{i}\right\}$ be a representation basis for $A$ defining the $Q_{i}$ as $\phi_{i}$, and with inverse $\Theta$. Let $\Phi^{*}=\left\{\phi_{i}^{*}\right\}$ be a representation basis for $A$, defining the $Q_{j}^{*}$ as $\phi_{j}^{*}$, and with inverse $\Theta^{*}$. Then:

(1) For each $Q_{j}^{*}$, there is $\alpha \in L_{Q}$ st $A+d_{\Phi}+d_{\Phi^{*}} \models \forall \bar{x}\left(Q_{j}^{*}(\bar{x}) \leftrightarrow \alpha\right)$.

(2) For each $Q_{i}$, there is $\beta \in L_{Q^{*}}$ st $A+d_{\Phi}+d_{\Phi^{*}} \models \forall \bar{x}\left(Q_{i}(\bar{x}) \leftrightarrow \beta\right)$.

Proof. One has definitions, in the definitional expansion $A+d_{\Phi}+d_{\Phi^{*}}$, of the symbols $Q_{i}$ and the $Q_{i}^{*}$ in terms of the $P_{i}$ symbols, as well as "inverse definitions" of the $P_{i}$ in terms of the $Q_{i}$ and the $Q_{i}^{*}$. One can then verify that there are definitions of the $Q_{i}$ in terms of the $Q_{i}^{*}$ and vice versa.

Theorem 14. Let an $L_{P}$-theory $T$ be given. Let $\Phi=\left\{\phi_{i}\right\}$ be a representation basis for $T$ defining the $Q_{i}$ as $\phi_{i}$, and with inverse $\Theta$. Let $\Phi^{*}=\left\{\phi_{i}^{*}\right\}$ be a representation basis for $T$, defining the $Q_{j}^{*}$ as $\phi_{j}^{*}$, and with inverse $\Theta^{*}$. Then:

(1) For each $Q_{j}^{*}$, there is $\alpha \in L_{Q}$ st $T+d_{\Phi}+d_{\Phi^{*}} \vdash \forall \bar{x}\left(Q_{j}^{*}(\bar{x}) \leftrightarrow \alpha\right)$.

(2) For each $Q_{i}$, there is $\beta \in L_{Q^{*}}$ st $T+d_{\Phi}+d_{\Phi^{*}} \vdash \forall \bar{x}\left(Q_{i}(\bar{x}) \leftrightarrow \beta\right)$.

Proof. Analogously to the previous result, one has definitions, in the definitional extension $T+d_{\Phi}+d_{\Phi^{*}}$, of the symbols $Q_{i}$ and the $Q_{i}^{*}$ in terms of the $P_{i}$ symbols, as well as "inverse definitions" of the $P_{i}$ in terms of the $Q_{i}$ and the $Q_{i}^{*}$. One can then verify that there are definitions of the $Q_{i}$ in terms of the $Q_{i}^{*}$ and vice versa.

\subsection{Criteria for Definitional Equivalence}

We extend the criteria for definitional equivalence (Theorem 8) with a sixth condition, now formulated in terms of "representation basis":

Theorem 15. The following are equivalent:

$$
\begin{gathered}
T_{1} \stackrel{\Phi}{\Theta} T_{2} . \\
T_{1}+d_{\Phi} \equiv T_{2}+d_{\Theta} . \\
\left(\tau_{\Theta} \tau_{\Phi}=1\right)_{T_{1}} \text { and } T_{2} \equiv D_{\Phi} T_{1} . \\
\left(T_{1}, \Phi\right) \leftrightarrow\left(T_{2}, \Theta\right) .
\end{gathered}
$$

$$
D_{\Phi}: \operatorname{Mod}\left(T_{1}\right) \leftrightarrow \operatorname{Mod}\left(T_{2}\right) \text { st, for any } A \models T_{1}, A \underset{\Theta}{\stackrel{\Phi}{\hookrightarrow}} D_{\Phi} A .
$$

(6) $\Phi$ is a representation basis for $T_{1}$ with inverse $\Theta$ and $T_{2} \equiv D_{\Phi} T_{1}$.

Proof. The equivalence of the first five criteria is stated in Theorem 8. To establish $(3) \Leftrightarrow(6)$, note that, using Theorem $10(1,3), \Phi$ is a representation basis for $T_{1}$ with inverse $\Theta$ if and only if $\left(\tau_{\Theta} \tau_{\Phi}=1\right)_{T_{1}}$. 
A (final) corollary is:

Theorem 16. Suppose $\Phi$ is a representation basis for $T_{1}$ with inverse $\Theta$. Then

$$
\begin{gathered}
T_{1} \stackrel{\Phi}{\stackrel{\Theta}{\leftrightarrow}} T_{2} . \\
\text { (2) } \operatorname{Mod}\left(T_{2}\right)=D_{\Phi}\left[\operatorname{Mod}\left(T_{1}\right)\right] .
\end{gathered}
$$

are equivalent.

Proof. For $(1) \Rightarrow(2)$, let us suppose first that $T_{1} \underset{\Theta}{\stackrel{\Phi}{\longrightarrow}} T_{2}$. Then this already implies that $\Phi$ is a representation basis for $T_{1}$ with inverse $\Theta$ and, furthermore, $T_{2} \equiv D_{\Phi} T_{1}$. So, by Theorem $12(5), D_{\Phi}\left[\operatorname{Mod}\left(T_{1}\right)\right]=\operatorname{Mod}\left(D_{\Phi} T_{1}\right)$. And since $T_{2} \equiv D_{\Phi} T_{1}$, we conclude that $\operatorname{Mod}\left(T_{2}\right)=D_{\Phi}\left[\operatorname{Mod}\left(T_{1}\right)\right]$.

For $(2) \Rightarrow(1)$, let us suppose instead that $\Phi$ is a representation basis for $T_{1}$ with inverse $\Theta$ and $\operatorname{Mod}\left(T_{2}\right)=D_{\Phi}\left[\operatorname{Mod}\left(T_{1}\right)\right]$. By Theorem 12(5), $D_{\Phi}\left[\operatorname{Mod}\left(T_{1}\right)\right]=\operatorname{Mod}\left(D_{\Phi} T_{1}\right)$. Hence, $\operatorname{Mod}\left(T_{2}\right)=\operatorname{Mod}\left(D_{\Phi} T_{1}\right)$. And so, $T_{2} \equiv$ $D_{\Phi} T_{1}$. And by condition (6) of Theorem 15, we conclude that $T_{1} \underset{\Theta}{\stackrel{\Phi}{\longrightarrow}} T_{2}$.

Thus, if $\Phi$ is a representation basis for $T_{1}$ with inverse $\Theta$ and $\operatorname{Mod}\left(T_{2}\right)=$ $D_{\Phi}\left[\operatorname{Mod}\left(T_{1}\right)\right]$, it follows that $T_{1} \stackrel{\Phi}{\stackrel{\Theta}{\longrightarrow}} T_{2}$. However, the counterexample from [1] shows that $\operatorname{Mod}\left(T_{2}\right)=D_{\Phi}\left[\operatorname{Mod}\left(T_{1}\right)\right]$ is too weak for this conclusion: the missing ingredient is that the defining set $\Phi$ be a representation basis for $T_{1}$ (with inverse $\Theta$ ).

\section{Theories and Basis Dependence}

There are several theories which have been carefully studied in mathematical logic - usually involving arithmetic and set theory - known to be definitionally equivalent.

But this is not a minor topic of narrow interest only to mathematical logicians. First, philosophers of science have long been interested in what constitutes either the empirical equivalence, or the full equivalence, of scientific theories. In the mid 70s, David Miller introduced the "language dependence" problem for theories of truthlikeness: the core of his argument being that changing which predicates are taken as primitive can affect comparisons of truthlikeness for false theories $([5-7])$. In metaphysics and epistemology, there is the famous example of "grue" and "bleen" predicates introduced by Nelson Goodman ([3]).

Second, there is an intuitive idea within mathematics and in physics of trying to eliminate dependence on "arbitrary choices", and that one prefers "basis independent" descriptions of mathematical objects. In a recent paper, Albert Visser notes:

The study of interpretability is, in part, about the escape from the tyranny of signature. Specific choices for the language are implementation artifacts introduced because, after all is said an done, we 
have to do things one way or another. Good mathematical properties of theories should be independent of these arbitrary choices. (Visser 2015 [8], p. 2 of preprint)

An example is the basis of a vector space $V$. Every vector space has a basis $\left\{\mathbf{e}_{a}\right\}$ and the space itself is reconstructed as the linear span of the basis. But any such basis itself is an "arbitrary parametrization" or "implementation" of $V$ and vectors in $V$ don't "care" what basis they are expanded relative to: given two bases $\left\{\mathbf{e}_{a}\right\}$ and $\left\{\mathbf{e}_{a}^{\prime}\right\}$, and some $\mathbf{v} \in V$, there are expansions:

$$
X^{a} \mathbf{e}_{a}=\mathbf{v}=Y^{a} \mathbf{e}_{a}^{\prime}
$$

Sometimes one basis is much more convenient to work with than others (e.g., certain matrices get diagonalized; certain operators take simpler forms). But these efficiency features have nothing to do with vector space itself.

Similarly, any particular chart $(U, \varphi)$ in a topological manifold is, in some sense, arbitrary. For the points in $M$ shouldn't really "care" what co-ordinates they are given in overlapping charts; say, charts $(U, \varphi)$ and $(V, \psi)$ where $U \cap V \neq$ $\varnothing$. And similarly, in the overlap region, there is an invertible mapping (a local homeomorphism) between the charts. If $M$ is a 3-manifold and I am told that $\varphi(x)=(0,0,0)$, then I have been told nothing intrinsic to $M$ itself; since any point $x \in M$ can be given those co-ordinates by some chart $\varphi$.

One wonders if structures and theories could be treated similarly. Here we have explained (Sect. 6) what it is for a set $\Phi=\left\{\phi_{i}\right\}_{i \in I}$ of formulas of a language $L$ to form a "representation basis" for an $L$-structure $A$ or for an $L$-theory $T$. The atomic formulas automatically do. But complex logical compounds may also form a representation basis for $A$ or $T$, so long as the corresponding system of definitions is "invertible". If the set $\Phi=\left\{\phi_{i}\right\}_{i \in I}$ is a representation basis, each primitive $P_{i}$ of the language $L$ can be given an explicit definition of the form $\forall \bar{x}\left(P_{i}(\bar{x}) \leftrightarrow \theta_{i}\right)$, where $\theta_{i}$ contains only new predicates $Q_{i}$ introduced via explicit definition to represent the $\phi_{i} \in \Phi$. These intertranslatable representation bases then provide "definitionally equivalent reparametrizations" of a structure $A$ or a theory $T$ (relative to $L$ ). ${ }^{11}$

Open Access. This article is licensed under a Creative Commons Attribution 4.0 International License, which permits use, sharing, adaptation, distribution and reproduction in any medium or format, as long as you give appropriate credit to the original author(s) and the source, provide a link to the Creative Commons licence, and indicate if changes were made. The images or other third party material in this article are included in the article's Creative Commons licence, unless indicated otherwise in a credit line to the material. If material is not included in the article's Creative Commons licence and your intended use is not permitted by statutory regulation or exceeds the permitted use, you will need to obtain permission directly from

${ }^{11} \mathrm{I}$ would like to acknowledge support from a grant from the National Science Centre in Kraków (NCN) (grant number 2018/29/B/HS1/01832). 
the copyright holder. To view a copy of this licence, visit http://creativecommons. org/licenses/by/4.0/.

Publisher's Note Springer Nature remains neutral with regard to jurisdictional claims in published maps and institutional affiliations.

\section{References}

[1] Andréka, H., Madarász, J.X., Németi, I.: Mutual definability does not imply definitional equivalence, a simple example. Math. Log. Quart. 51(6), 591-597 (2005)

[2] Andréka, H., Németi, I.: Definability Theory Course Notes. https://old.renyi.hu/ pub/algebraic-logic/DefThNotes0828.pdf. (2014)

[3] Goodman, N.: Fact, Fiction, and Forecast. Athlone Press, London (1954)

[4] Ketland, J.: Bases for structures and theories I. Logica Universalis 14, 357-381 (2020)

[5] Miller, D.W.: On popper's qualitative theory of verisimilitude. Br. J. Philos. Sci. 25, 166-177 (1974)

[6] Miller, D.W.: The accuracy of predictions. Synthese 30, 159-191 (1975)

[7] Miller, D.W.: The distance between constituents. Synthese 38, 197-212 (1978)

[8] Visser, A.: Extension and Interpretability. Preprint, (2015)

Jeffrey Ketland

Institute of Philosophy

University of Warsaw

Krakowskie Przedmieście 3

00-927 Warszawa

Poland

e-mail: jeffreyketland@gmail.com

Received: May 26, 2020.

Accepted: September 13, 2020. 